\title{
Article \\ Conceptual Framework for Quantum Affective Computing and Its Use in Fusion of Multi-Robot Emotions
}

\author{
Fei Yan ${ }^{1}\left(\mathbb{D}\right.$, Abdullah M. Iliyasu ${ }^{2,3, *}$ (D) and Kaoru Hirota ${ }^{3,4}(\mathbb{D}$ \\ 1 School of Computer Science and Technology, Changchun University of Science and Technology, \\ Changchun 130022, China; yanfei@cust.edu.cn \\ 2 College of Engineering, Prince Sattam Bin Abdulaziz University, Al-Kharj 11942, Saudi Arabia \\ 3 School of Computing, Tokyo Institute of Technology, Yokohama 226-8502, Japan; hirota@jsps.org.cn \\ 4 School of Automation, Beijing Institute of Technology, Beijing 100081, China \\ * Correspondence: a.iliyasu@psau.edu.sa
}

Citation: Yan, F.; Iliyasu, A.M.

Hirota, K. Conceptual Framework for

Quantum Affective Computing and Its Use in Fusion of Multi-Robot Emotions. Electronics 2021, 10, 100. http:/ / doi.org/10.3390/ electronics 10020100

Received: 20 November 2020 Accepted: 1 January 2021 Published: 6 January 2021

Publisher's Note: MDPI stays neutral with regard to jurisdictional clai$\mathrm{ms}$ in published maps and institutional affiliations.

Copyright: (C) 2021 by the authors. Licensee MDPI, Basel, Switzerland. This article is an open access article distributed under the terms and conditions of the Creative Commons Attribution (CC BY) license (https:// creativecommons.org/licenses/by/ $4.0 /)$.

\begin{abstract}
This study presents a modest attempt to interpret, formulate, and manipulate the emotion of robots within the precepts of quantum mechanics. Our proposed framework encodes emotion information as a superposition state, whilst unitary operators are used to manipulate the transition of emotion states which are subsequently recovered via appropriate quantum measurement operations. The framework described provides essential steps towards exploiting the potency of quantum mechanics in a quantum affective computing paradigm. Further, the emotions of multi-robots in a specified communication scenario are fused using quantum entanglement, thereby reducing the number of qubits required to capture the emotion states of all the robots in the environment, and therefore fewer quantum gates are needed to transform the emotion of all or part of the robots from one state to another. In addition to the mathematical rigours expected of the proposed framework, we present a few simulation-based demonstrations to illustrate its feasibility and effectiveness. This exposition is an important step in the transition of formulations of emotional intelligence to the quantum era.
\end{abstract}

Keywords: affective computing; quantum computing; artificial intelligence; quantum emotion; emotion fusion; social robots

\section{Introduction}

Affective computing is the study and development of systems and devices that can recognise, interpret, process, and simulate affective phenomena [1]. While some developments in the field may be traced back to early philosophical inquiries into emotion [2], the more modern branch of computer science originated with Picard's extensive works in [3] and flourished into an interdisciplinary field spanning computer science, psychology, and cognitive science [4]. One of the motivations for the field is the ability to ingrain emotional intelligence into machines so that they could interpret emotional states of humans and adapt its behaviour to them as well as providing an appropriate response to those emotions [5].

In artificial intelligence, interaction between artificial systems and their users is improved by making such systems not just intelligent but also emotionally sensitive [6]. Research in affective computing is encumbered by fundamental issues of seriality in the computational hardware whereas the human brain fundamentally works differently [7]. The physical make-up of the human brain (often divided into rational and emotional brains) is such that information is simultaneously processed rationally and emotionally, while in the area of social robotics, this conundrum clumsiness in a robot's behaviour, i.e., conventional computers cannot effectively process information in parallel. This would suggest that incongruity of using such frameworks to model the human affective system, which is known to be capable of manifesting concurrent emotional experiences (for example, happy and sad) [8]. 
When all is said and done, the massive amount of data that will be generated by future sensors combined with the rising complexity associated with controlling, planning, interacting, and reasoning systems, as well as the fact that future robots will operate in all kinds of environments, especially those to facilitate interactions with humans. This will be further aggravated when these robots are connected via internet of things (IoT) frameworks. All these indicate the need for enormous computing resources for future robotic systems. Meanwhile, quantum systems are credited with astounding capabilities ascribed to its properties of entanglement, superposition, and parallelism [9]. Therefore, there is a consensus cum growing interest in harnessing quantum algorithms, quantum sensors, and quantum controls for important roles in future robotics and automated integrated systems.

Exploiting the overwhelming superiority that quantum computing offers [10], numerous studies exploring the use of quantum mechanisms in affective computing have been proposed. For example, in [11], Schwartz et al. enthused the need for current neuropsychology to incorporate the mathematics of quantum physics in accounting for human observational bias in the measurement of physical properties of the human brain. Building on his previous efforts, Aerts asserted that a number of quantum mechanical principles are at the origin of specific effects in cognition, and based on which a general hypothesis about the quantum structure of human thought was investigated [12,13]. Similarly, Narens et al. argued that psychology may not be effective in transferring contextual dialogues into probabilistic models but found solace in the belief that quantum probability theory could better handle the dynamics of contextual impact on behaviours [14].

Following these insights, in [15], Lukac et al. provided a quantum mechanical model of robot-specific emotions based on quantum cellular automata, where a robot is described as a set of functional agents each acting on behalf of its own emotional function. Years later, Raghuvanshi et al. proposed concepts that apply quantum circuits to model fuzzy sets, which birthed a new method to model emotional behaviour for a humanoid robot [16]. More recently, in [17], Yan et al. proposed a framework portraying emotion transition and fusion, which is encoded as quantum emotion space with three entangled qubit sequences to locate an emotion point within a three-dimensional emotion space. They surmised that such a framework facilitates easier tracking of emotion transitions over different intervals in the emotion space. However, as a drawback, their definition of emotion relies on the two qubit sequences, which is insufficient when more sophisticated emotions are desired.

To summarise, to date, efforts to integrate quantum mechanics into different aspects of affective computing can be divided into two groups: (1) applications that exploit some of the properties responsible for the potency of quantum computing algorithms as tools to improve some available affective computing tasks, and (2) applications that derive their inspiration from the expectation that quantum computing hardware will soon be physically realised and, hence, such studies focus on extending affective computing tasks to the quantum computing framework. These directions motivate two fundamental questions regarding the notion of quantum emotion: First, what advantages do the resulting quantum emotion offer us? And, second, how does this "quantumness" relate to possible descriptions of future quantum robots [18]?

While there may be other questions, answering these two questions as well as exploring the integration of some of the remarkable advances recorded in quantum machine learning [19], quantum artificial intelligence [20], and quantum image processing [21] provide few of the other motivations for this study. For the first question, the use of quantum mechanics in describing emotion presupposes the potential use of parallel processing power and demand lower computing resources since quantum computing offers exponential storage and speed up relative to the required number of qubits as well as the sufficient (polynomial) number of CNOT gates that are needed to manipulate quantum emotion. Regarding the second question, by conceptualising a quantum robot as a mobile quantum system that includes an on-board quantum computer and its required ancilliary systems [22], it is reasonable to imagine that quantum robots will carry out tasks whose goals include specified changes and adjustments to emotions in conformity with the state 
of the environment or even carrying out measurements on the environment itself. Similarly, integrating quantum mechanics into descriptions of robot emotion supports potential development of quantum robots as well as its use to manipulate classical robot devices to perform advanced emotion-related actions [23].

Consequent upon the foregoing survey, in this study, we propose a conceptual framework for quantum affective computing (QAC) as a quantum computing unit within a quantum robot system (defined here as a quantum robot that includes many quantum computing units and each unit is supposed to accomplish specific tasks and exchange of information with each other). While affective computing is defined as presented at the beginning of this section, the primary purpose of QAC is to enhance the capacity for storing, processing, and retrieving the affective information in the human-robot interaction either by transitioning from traditional to quantum paradigms (e.g., as used for quantum robots) or by complementing traditional procedures with quantum techniques (e.g., the quantum machine learning algorithm mentioned earlier). A QAC unit is one ingrained with the capability to receive external stimulus, and its manipulation as quantum signals which can motivate (or compel) the quantum robot to carry out some actions on its environment, such as emotion expression and path planning. The first step in accomplishing this is formulating a representation for emotion within the precepts of quantum mechanics and then realising its manipulation and fusion for different applications in quantum-based affective computing.

The remainder of the study is organised as follows. In Section 2, we present some of quantum properties and propose our framework for QAC. In Section 3, we define the quantum representation of robot emotion and discuss their basic transitions. In Section 4, we discuss the intrigues involved infusing multiple robot emotions and illustrate both the fusion and retrieving procedures.

\section{Conceptual Framework of Quantum Affective Computing}

As discussed earlier, affective computing aims to leverage the capability of computing technologies to recognise, understand, represent, and adapt human's emotion towards building harmonious human-robot environment [24]. As a prelude to our discussion on QAC, in this section, we highlight a brief background on quantum computing with focus on its bewildering properties of superposition and entanglement, and discuss how such properties to imitate human behaviour as well as working mechanisms the brain uses to process emotions. Based on this background, later in the section, we propose our conceptual framework of QAC.

\subsection{Quantum Effects in Human Behaviour}

Analogous to the fundamental concept of classical computation, the bit, a quantum bit (or qubit) is the smallest unit of information in the quantum system [10]. The difference between bits and qubits lies in the latter's ability to propagate in a superposition state, which can be described as a unit vector in two-dimensional Hilbert space. As shown in Figure 1a, the vector can always be written as $|\psi\rangle=\alpha|\uparrow\rangle+\beta|\rightarrow\rangle$, where $|\uparrow\rangle$ and $|\rightarrow\rangle$ are orthogonal basis states and $\alpha$ and $\beta$ are complex numbers for probability amplitudes. The probabilities for $|\psi\rangle$ to be in the $|\uparrow\rangle$ and $|\rightarrow\rangle$ states are, respectively, $|\alpha|^{2}$ and $|\beta|^{2}$, where $|\alpha|^{2}+|\beta|^{2}=1$. Geometrically, this can be interpreted as the condition that the qubit's state is normalized to length 1 . If we let $|\uparrow\rangle=|0\rangle$ and $|\rightarrow\rangle=|1\rangle$, then $|\psi\rangle=\alpha|0\rangle+\beta|1\rangle$, so the states $|0\rangle$ and $|1\rangle$ are known as computational basis states, and they form an orthonormal basis for this vector space. 


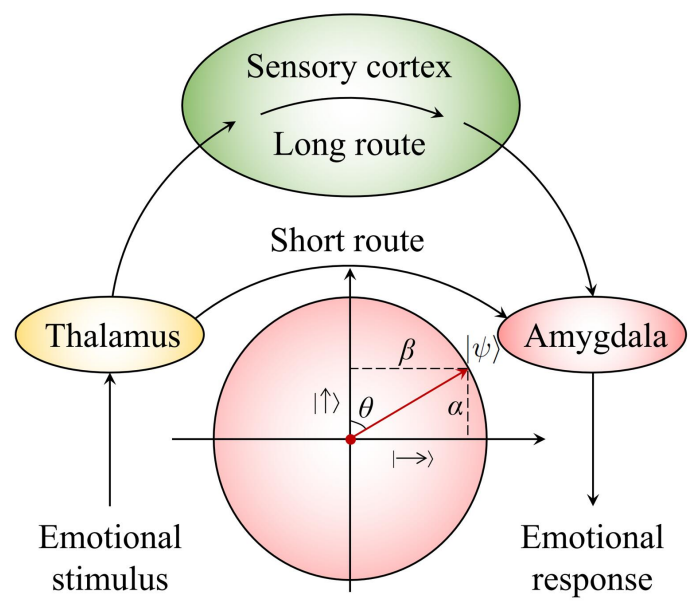

(a)

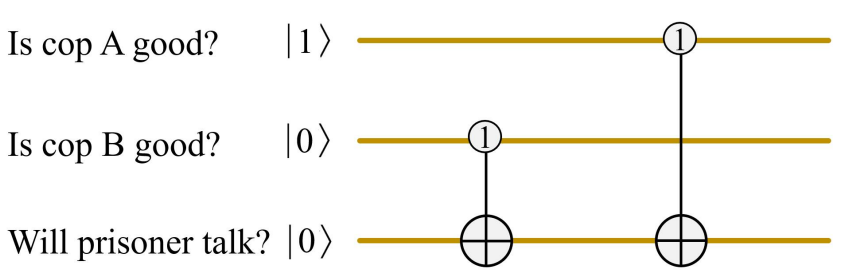

Is Alice happy?

Is Bob happy?

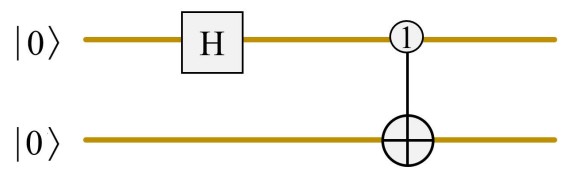

(b)

Figure 1. Illustration of (a) a qubit's superposition state and (b) quantum logic circuits (as explained in the text).

As espoused via the mechanism of human emotional response (depicted in Figure 1a), when a stimulus is received from the outside world, there are usually two routes to transmit information to the brain and make corresponding responses. They are the short route from the thalamus to amygdala directly in emotional brain, and the long route from the thalamus to amygdala through the cortex in rational brain. Absence of information processing by the cortex in the short route makes it faster to execute, which ensures the rapid response to external stimulation. In contrast, stimulus information via the long route is processed by the sensory cortex, which is conducive to the control of emotion and the adoption of appropriate coping style. The main function of the amygdala in the brain is to produce emotions (which are adapted to various external information introduced into the neocortex of the brain). Similar to this, in therobotic aspect of affective computing, i.e., receiving the stimulus and after the processing to make the corresponding response, received stimuli are processed to determine the best response. Consequently, interpretations of affective computing from the quantum viewpoint, i.e., quantum affective computing (QAC), entails assimilating the mechanism of the amygdala and its role in generating and responding to emotions within the precepts of quantum mechanics [25], for example, the state the amygdala is in can be represented as a vector (red arrow in Figure 1a) of the two eigenstates in a Bloch sphere [8].

In quantum computing, a quantum circuit is a computation model in which a computation is a sequence of quantum logic gates (or simply quantum gates) [26]. Quantum gates are represented by unitary matrices described as an operator $U_{f}$ acting on a quantum state (a unitary matrix) then, $U_{f} U_{f}^{+}=I$, where $U_{f}^{+}$is the conjugate-transpose matrix of $U_{f}$ and $I$ is an identity matrix as presented in Figure 2. Since the unitary transform is reversible, it is logical that using quantum gates composed of the $U_{f}$ and $U_{f}^{+}$transforms each of the input and output states can be reversed. Mathematically, this can be expressed via $U_{f}|x\rangle=|f(x)\rangle$ and $U_{f}^{\dagger}|f(x)\rangle=|x\rangle$. It is noteworthy that quantum parallelism allows quantum computers to simultaneously evaluate a function $f(x)$ for many values of $x$ [27]. Using such a property, the fusion of quantum emotions (i.e., mixed emotions) for multi-robots and their emotion processes can be manipulated simultaneously, i.e., in parallel. 


\begin{tabular}{|c|c|c|c|c|}
\hline Gate & Equation & Matrix & Transform & Notation \\
\hline $\begin{array}{l}\text { Identity } \\
\text { (I) }\end{array}$ & $\boldsymbol{I}=|0\rangle\langle 0|+| 1\rangle\langle 1|$ & $\left(\begin{array}{ll}1 & 0 \\
0 & 1\end{array}\right)$ & $\begin{array}{l}\boldsymbol{I}|0\rangle=|0\rangle \\
\boldsymbol{I}|1\rangle=|1\rangle\end{array}$ & - \\
\hline $\begin{array}{c}\text { Pauli }-X \\
(X \text { or NOT })\end{array}$ & $\boldsymbol{X}=|0\rangle\langle 1|+| 1\rangle\langle 0|$ & $\left(\begin{array}{ll}0 & 1 \\
1 & 0\end{array}\right)$ & $\begin{array}{l}X|0\rangle=|1\rangle \\
X|1\rangle=|0\rangle\end{array}$ & \\
\hline $\begin{array}{l}\text { Hadamard } \\
\qquad(\boldsymbol{H})\end{array}$ & $\boldsymbol{H}=\frac{|0\rangle+|1\rangle}{\sqrt{2}}\langle 0|+\frac{|0\rangle-|1\rangle}{\sqrt{2}}\langle 1|$ & $\frac{1}{\sqrt{2}}\left(\begin{array}{cc}1 & 1 \\
1 & -1\end{array}\right)$ & $\begin{aligned} H|0\rangle & =\frac{1}{\sqrt{2}}(|0\rangle+|1\rangle) \\
H|1\rangle & =\frac{1}{\sqrt{2}}(|0\rangle-|1\rangle)\end{aligned}$ & - \\
\hline $\begin{array}{l}\text { Controlled- } \\
\text { NOT } \\
(\text { CNOT) }\end{array}$ & CNOT $=|0\rangle\langle 0|\otimes \boldsymbol{I}+| 1\rangle\langle 1| \otimes \boldsymbol{X}$ & $\left(\begin{array}{llll}1 & 0 & 0 & 0 \\
0 & 1 & 0 & 0 \\
0 & 0 & 0 & 1 \\
0 & 0 & 1 & 0\end{array}\right)$ & $\begin{array}{l}\text { CNOT }|00\rangle=|00\rangle \\
\text { CNOT }|01\rangle=|01\rangle \\
\text { CNOT }|10\rangle=|11\rangle \\
\text { CNOT }|11\rangle=|10\rangle\end{array}$ & \\
\hline $\begin{array}{c}\text { Toffoli } \\
\text { ( } \boldsymbol{T} \text { or } \\
\text { CCNOT) }\end{array}$ & $\begin{aligned} \mathbf{T} & =|0\rangle\langle 0| \otimes \boldsymbol{I} \otimes \boldsymbol{I} \\
& +|1\rangle\langle 1| \otimes \mathbf{C N O T}\end{aligned}$ & $\left(\begin{array}{llllllll}1 & 0 & 0 & 0 & 0 & 0 & 0 & 0 \\
0 & 1 & 0 & 0 & 0 & 0 & 0 & 0 \\
0 & 0 & 1 & 0 & 0 & 0 & 0 & 0 \\
0 & 0 & 0 & 1 & 0 & 0 & 0 & 0 \\
0 & 0 & 0 & 0 & 1 & 0 & 0 & 0 \\
0 & 0 & 0 & 0 & 0 & 1 & 0 & 0 \\
0 & 0 & 0 & 0 & 0 & 0 & 0 & 1 \\
0 & 0 & 0 & 0 & 0 & 0 & 1 & 0\end{array}\right)$ & $\begin{array}{l}\mathbf{T}|000\rangle=|000\rangle, \mathbf{T}|001\rangle=|001\rangle \\
\mathbf{T}|010\rangle=|010\rangle, \mathbf{T}|011\rangle=011\rangle \\
\mathbf{T}|100\rangle=|100\rangle, \mathbf{T}|101\rangle=|101\rangle \\
\mathbf{T}|10\rangle=|11\rangle, \mathbf{T}|11\rangle=\| 110\rangle\end{array}$ & \\
\hline
\end{tabular}

Figure 2. Properties of commonly used basic quantum gates.

The notion of quantum circuits that correspond to affective behaviour is introduced by Raghuvanshi et al. in [16], i.e., to employ EPR (Einstein-Podolsky-Rosen) circuits to set qubits into an entangled state to influence the human behaviour. For instance, the first circuit in Figure $1 \mathrm{~b}$ presents the behaviour of the prisoner. The prisoner is willing to talk when one cop is good and one cop is bad (i.e., utilises a strategy combing both "hard " and "soft" means), otherwise, his willingness level does not chance, i.e., the prisoner will never talk. Further, considering another example we show the behaviour between Alice and Bob using the second circuit in Figure $1 \mathrm{~b}$. If we define 1 as indicating happy and 0 as unhappy, after the quantum gate operation, the behaviour between Alice and Bob is entangled. So if both Alice and Bob are unhappy, so the input is $|00\rangle$ and output becomes $\frac{1}{\sqrt{2}}|00\rangle+\frac{1}{\sqrt{2}}|11\rangle$, there is one half probability that they will talk to each other and still be unhappy, and there is one half probability that they will talk to each other (and perhaps they comfort and encourage mutually), so they will both become happy.

The final step in any quantum computation task is the measurement operation, which converts the transformed quantum information into the classical information in form of probability distributions [28]. Even though a qubit can represent many states, when it is observed, the measurement results can only be either 0 or 1 , with a certain probability. This operation converts a single qubit state $|\psi\rangle=\alpha|0\rangle+\beta|1\rangle$ into a probabilistic classical bit, which is 0 with probability $|\alpha|^{2}$ or 1 with probability $|\beta|^{2}$. In affective computing that is built on quantum mechanics, quantum properties allow for existence of multiple emotions in the Hilbert space represented by the phases. After measurements, however, only one emotion will be observed. This is analogous to the human behaviour where in the human mind, there are numbers of emotions, but the actions typically represent one emotion [16].

\subsection{Quantum Framework for Affective Computing}

In [29], Dong et al. presented the structural background of a quantum robot including description of its fundamental components and frameworks for multi-quantum computing units (MQCU), quantum actuator, and information acquisition units as presented in 
Figure 3. Based on this structure, a quantum robot uses the information acquisition units to perceive its environment and acquire information, and then send sensing information to the MCQU. An MQCU is made up of many quantum computing units (QCUs) and, as well as accomplishing specified tasks, QCU exchanges information with each other through a quantum bus (which may be a refreshable entanglement resource [30]). The MQCU processes the information and generates new signals or learning control algorithms that are sent to the quantum actuator until the task is accomplished. Finally, the quantum actuator receives information from the MQCU and carries out specified actions on its environments, which is regarded as an interaction channel between a quantum robot and its environments (i.e., in addition to the information acquisition units).

Moreover, quantum robots have communication interfaces to exchange information with distant mainframes or other quantum robots, which can constitute a multi quantum robot system as presented in Figure 4. In external communication, the spectacular properties of high channel capability, perfect security and efficient teleportation in quantum communication can be used to exchange information [29]. These features could also be used to facilitate the fusion of emotion for multi-robots in a communication scenario, which will be discussed later in Section 4 .

Guided by the intuition highlighted above, we present the framework of QAC which is regarded as a unit within the MQCU. This framework is envisioned as a QCU that facilitates exchange of information with other components of the MQCI. To encapsulate this intuition, the proposed framework is built on the use of several hypothetical quantum devices, such as emotion generator (EG), emotion controller (EC), emotion sensor (ES), and emotion reader (ER), as required to achieve the initialisation, manipulation, and retrieval of emotions. As presented in Figure 3, the EG initialises the quantum emotion by transforming the quantum system from its initial state (i.e., an array of $|0\rangle$ states) to a specified emotion state. Usually, this information consists of both quantum and classical information, which precludes frequent interactions with the outside world since quantum measurement destroys the quantum system. This pitfall can be avoided by adopting either of two viewpoints to affective computing. First, from the point of view of physiology, we may record the human body's various physiological parameters by means of various measurements and sensors, e.g., data on human motions, facial expressions, pulses, and brain waves. Subsequently, all such information can be used to compute the human's emotional status. Second, based on the psychological viewpoint, where different sensors can be used to receive and process the data. According to this, information to compute the robot's instantaneous emotional state can be computed. The ES receives the stimulus (such as an environment with music playing [31]) received from the external world into a set of unitary operations in a format that is amenable to manipulation using quantum resources. In this regard, a quantum sensor is a kind of microstructural sensor, that is designed by applying quantum effect. To detect faint classical signals (external stimulus), as presented in [29], two types of quantum sensors, superconduction quantum interference device sensor [32] and quantum well Hall sensor [33] can be considered. The EC transforms and controls the quantum emotion signal using quantum operators, i.e., quantum gates. The quantum controller acts as the connection between the current emotion signals and the external stimulus (quantum operators) to produce the desiredor undesired signal processing outcomes. Usually, the quantum controllers are a combination of quantum gates that can the rapidly developing quantum control theory [34] in its design. Finally, the ER retrieves the quantum emotional state of the robot by applying quantum measurement operations. This could be a pure quantum apparatus or a hybrid semiclassical apparatus capable of reading quantum information [35]. Final choice of either would depend on how we intend to use the produced quantum signals. For example, it may be used in the quantum system to direct an actuator to carry out corresponding actions on external environments or a readout to control some classical device. 


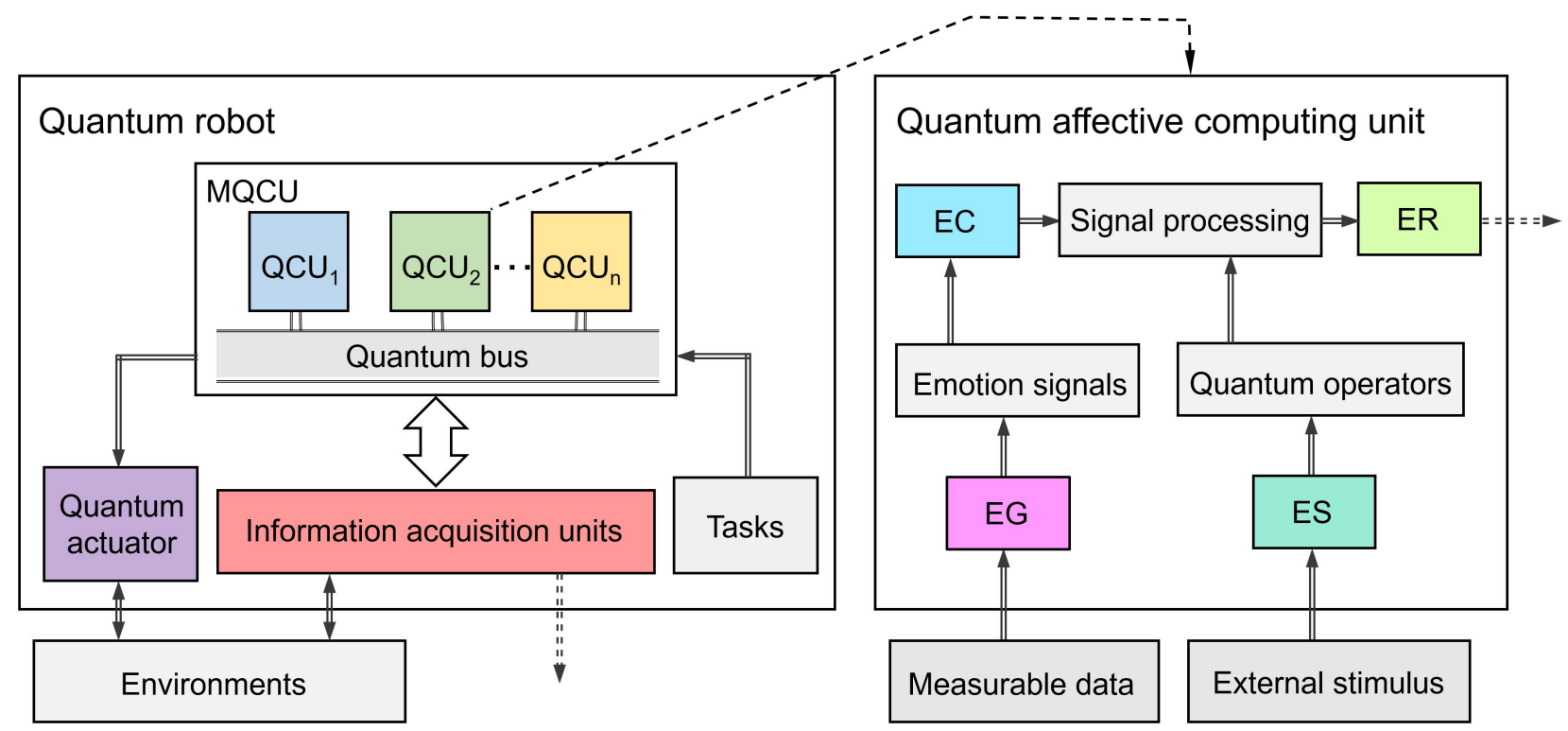

Figure 3. Quantum robot system showing three interacting components and framework depicting quantum affective computing (QAC) unit.

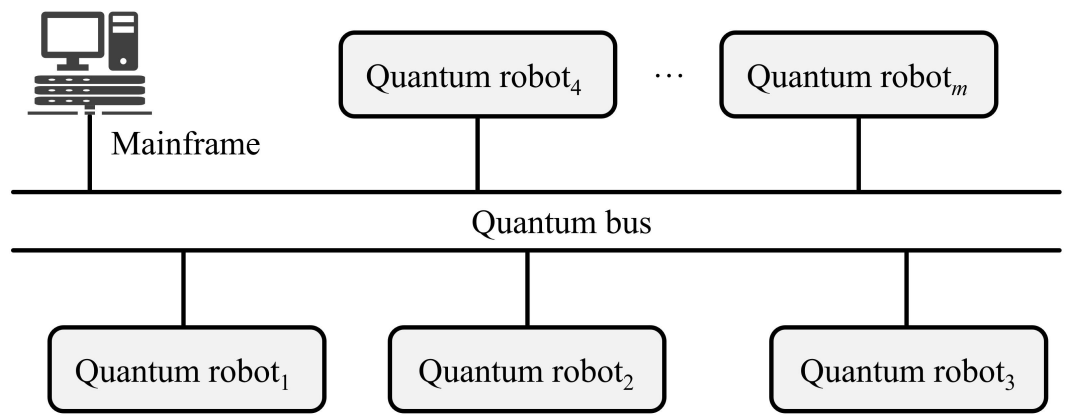

Figure 4. Illustration of quantum system composed of multi-robots in a communication scenario (figure adapted from [29]).

\section{Quantum Representation for Robot Emotion}

A traditional two-dimensional pleasure-arousal (PA) plane is characterised by a pleasure-displeasure and an arousal-sleep plane that indicate both the specific emotions and general features common to many different emotions [36]. In addition, unlike the expression of sensitive feelings in human communication, discussions about robot emotion are relatively straightforward. Therefore, we modify the original PA plane in [36] and represented in the form depicted in Figure 5a. As seen in that figure, there are four quadrants that are labelled as "Excitement", "Distress", "Depression", and "Contentment". Since our study is focused on the robot emotion, each quadrant of the modified PA plane can be temporarily roughly divided into two key emotions illustrated in the figure.

We adopt the PA plane with 8 emotions (using angles in different intervals) in the four quadrants as shown in Figure $5 \mathrm{~b}$. The colours are selected according to established psychological interpretations, and the colour information corresponds to the selected emotion for visualisation [37]. It is noteworthy that researchers have divergent opinions regarding the use of colour coding in descriptions of emotions. Its use here is for clarity in visualisation of the emotions. The actual parameters used to interpret the emotions are bifurcations represented by the angles in Figure $5 b$, which, as already explained, are encoded as quantum superposition states. The type of emotions is chosen according to the coordinate values in the original PA plane. Since angle values in the range 0 to $2 \pi$ are continuous, by this spectrum, we can add more intervals (representing more emotions) according to the situation. 


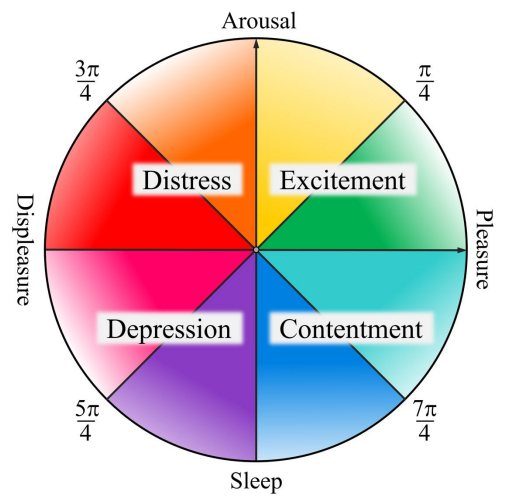

(a)

\begin{tabular}{|c|c|c|c|c|c|c|c|}
\hline Green & Yellow & Orange & Red & Pink & Purple & Blue & Indigo \\
\hline \multicolumn{2}{|c|}{$\left[0, \frac{\pi}{2}\right)$} & \multicolumn{2}{|c|}{$\left[\frac{\pi}{2}, \pi\right)$} & \multicolumn{2}{|c|}{$\left[\pi, \frac{3 \pi}{2}\right)$} & \multicolumn{2}{|c|}{$\left[\frac{3 \pi}{2}, 2 \pi\right)$} \\
\hline \multicolumn{2}{|c|}{ Excitement } & \multicolumn{2}{|c|}{ Distress } & \multicolumn{2}{|c|}{ Depression } & \multicolumn{2}{|c|}{ Contentment } \\
\hline$\left[0, \frac{\pi}{4}\right)$ & {$\left[\frac{\pi}{4}, \frac{\pi}{2}\right)$} & {$\left[\frac{\pi}{2}, \frac{3 \pi}{4}\right)$} & {$\left[\frac{3 \pi}{4}, \pi\right)$} & {$\left[\pi, \frac{5 \pi}{4}\right)$} & {$\left[\frac{5 \pi}{4}, \frac{3 \pi}{2}\right)$} & {$\left[\frac{3 \pi}{2}, \frac{7 \pi}{4}\right)$} & {$\left[\frac{7 \pi}{4}, 2 \pi\right)$} \\
\hline Happiness & Surprise & Fear & Anger & Sadness & Boredom & Relaxation & Expectation \\
\hline
\end{tabular}

(b)

Figure 5. Description of (a) modified pleasure-arousal (PA) plane showing four quadrants and (b) 16 emotions as well as colour and angle intervals for the planes.

Recalling the equation $|\psi\rangle=\alpha|0\rangle+\beta|1\rangle$ and its the discussion in Section 2, we let $\alpha=\cos \theta$ and $\beta=\sin \theta, \theta \in[0,2 \pi)$, then $|\psi\rangle=\cos \theta|0\rangle+\sin \theta|1\rangle$. Further, if we let $\xi=\theta / 4$, then $\xi \in\left[0, \frac{\pi}{2}\right]$. We do this because the trigonometric function is monotonically changing in the period. In this case, we need to proportionally map the emotions in the range 0 to $2 \pi$ to a new interval of $[0, \pi / 2]$. Since the angles in these two intervals are continuous, bijection is a useful tool to accomplish the desired transformation. In other words, we can map all the eight types of emotions via the angles in the interval of $[0, \pi / 2]$. Consequently, mathematically, the quantum emotion of a robot can be expressed as

$$
|E(\xi)\rangle=\cos \xi|0\rangle+\sin \xi|1\rangle,
$$

where $\xi \in\left[0, \frac{\pi}{2}\right]$ is regarded as the emotional parameter (or variable); $|0\rangle$ and $|1\rangle$ are 2-D computational basis quantum states. Following this formalism, it is important to discuss how a desired quantum emotion state of a robot can be generated. Given an angle $\xi \in\left[0, \frac{\pi}{2}\right]$, a rotation matrix (rotation around the $y$-axis of a Bloch sphere by angle $2 \xi$ ) is defined as

$$
R_{y}(2 \xi)=\left(\begin{array}{cc}
\cos \xi & -\sin \xi \\
\sin \xi & \cos \xi
\end{array}\right),
$$

which transforms the computational basis state into the desired quantum emotion state, expressed as

$$
R_{y}(2 \xi)|0\rangle=\left(\begin{array}{cc}
\cos \xi & -\sin \xi \\
\sin \xi & \cos \xi
\end{array}\right)\left(\begin{array}{l}
1 \\
0
\end{array}\right)=\cos \xi|0\rangle+\sin \xi|1\rangle .
$$

Now, to transform the emotional state, we consider several single quantum gates and apply them on the quantum emotion state in Equation (1). $X$ gate exhibits the property that $X|0\rangle=|1\rangle$ and $X|1\rangle=|0\rangle$; so, when it is applied on an emotion state, its impact can be expressed as

$$
X(|E(\xi)\rangle)=\left(\begin{array}{cc}
0 & 1 \\
1 & 0
\end{array}\right)\left(\begin{array}{c}
\cos \xi \\
\sin \xi
\end{array}\right)=\left|E\left(\frac{\pi}{2}-\xi\right)\right\rangle,
$$

where $|E(\xi)\rangle$ is the emotion state as defined in Equation (1). The function of the $X$ gate is like the emotion inversion operation that flips every given emotion.

Mathematically, the $Z$ gate has the properties that $Z|0\rangle=|0\rangle$ and $Z|1\rangle=-|1\rangle$; so, when it is applied on the emotion state, we have the transformation:

$$
Z(|E(\xi)\rangle)=\left(\begin{array}{cc}
1 & 0 \\
0 & -1
\end{array}\right)\left(\begin{array}{c}
\cos \xi \\
\sin \xi
\end{array}\right)=|E(-\xi)\rangle,
$$


whose function is the negation operation, i.e., to change the sign of the angle. On the QAC framework, when combined with other transformations, this operation produces many useful outcomes. This will be discussed later.

The $H$ gate executes the transformation $H|0\rangle=\frac{1}{\sqrt{2}}(|0\rangle+|1\rangle)$ and $H|1\rangle=\frac{1}{\sqrt{2}}(|0\rangle-$ $|1\rangle)$. Therefore, when it is applied on the emotion sate the outcome can be expressed as

$$
H(|E(\xi)\rangle)=\frac{1}{\sqrt{2}}\left(\begin{array}{cc}
1 & 1 \\
1 & -1
\end{array}\right)\left(\begin{array}{c}
\cos \xi \\
\sin \xi
\end{array}\right)=\left|E\left(\frac{\pi}{4}-\xi\right)\right\rangle .
$$

As we shall see later, this operation can be used to neutralise (i.e., cancel out) an emotional state.

The general form resulting from the combination of the three transformations (i.e., $X$, $\mathrm{Z}$, and $H$ ) can be expressed as a unitary matrix in the form:

$$
C(2 \eta)=\left(\begin{array}{cc}
\cos \eta & \sin \eta \\
\sin \eta & -\cos \eta
\end{array}\right)
$$

where $\eta \in[0, \pi / 2]$. When applied on the emotion state of a robot, the $C(2 \eta)$ operator transforms the emotion to a state:

$$
C(2 \eta)(|E(\xi)\rangle)=\left(\begin{array}{cc}
\cos \eta & \sin \eta \\
\sin \eta & -\cos \eta
\end{array}\right)\left(\begin{array}{c}
\cos \xi \\
\sin \xi
\end{array}\right)=|E(\eta-\xi)\rangle .
$$

This operation transforms a given emotion to a new one, within the bound encoded $\xi$ and $\eta-\xi$, respectively. As inferred in Equation (5), combining this operation with the $Z$ gate changes the emotion to a new state whose angles is $\eta+\xi$. Moreover, transformations, $X, Z$, and $H$, are the special cases of $C(2 \eta)$ in which $\eta$ is equal to $\pi / 2,0$, and $\pi / 4$, respectively.

Furthermore, by using the definition of $C(2 \eta)$, that of the rotation matrix in Equation (2) and the property of the Pauli $Z$ matrix, we obtain the useful operation exhibited as

$$
C(2 \eta)=\left(\begin{array}{cc}
\cos \eta & -\sin \eta \\
\sin \eta & \cos \eta
\end{array}\right)\left(\begin{array}{cc}
1 & 0 \\
0 & -1
\end{array}\right)=R_{y}(2 \eta) \times Z
$$

An implication arising from Equation (9) is that trivial tasks to show increase or decrease of emotion from $\xi$ to $\xi+\eta$ or from $\xi$ to $\xi-\eta$ can be achieved by using $R_{y}(2 \eta)$ or $R_{y}(-2 \eta)$, respectively. These two operations are expressed by

$$
R_{y}(2 \eta)|E(\xi)\rangle=|E(\xi+\eta)\rangle
$$

and

$$
R_{y}(-2 \eta)|E(\xi)\rangle=|E(\xi-\eta)\rangle .
$$

The matrix $R_{y}(2 \eta)$ has unit determinant and exhibits the following properties:

$$
\begin{aligned}
& R_{y}(2 \eta) \times R_{y}(-2 \eta)=I, \\
& R_{y}(-2 \eta)=X \times R_{y}(2 \eta) \times X, \\
& R_{y}\left(2 \eta_{1}+2 \eta_{2}\right)=R_{y}\left(2 \eta_{1}\right) \times R_{y}\left(2 \eta_{2}\right) .
\end{aligned}
$$

Following the above mathematical formalism, in the quantum circuit model of computation, we can use circuit elements can be used to illustrate the execution of the same operations highlighted earlier. The $R(2 \eta)$ operation can be realised using a combination of two rotation gates and two controlled-NOT (CNOT) gates as presented in Figure 6a. Here, when the control wire has an input $|0\rangle$ state, the operation $R(\eta) \times R(-\eta)$, which is equal to the identity operation is executed. This translates to the first property in Equation (12). Therein, when the control wire is in the $|1\rangle$ state, the operation $R(\eta) \times X \times R(-\eta) \times X$ is equal to the $R(2 \eta)$ that is executed. An extended version of this operation with multiple control conditions is presented in Figure $6 \mathrm{~b}$. 

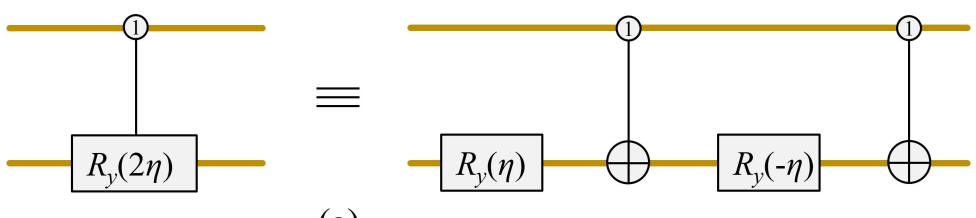

(a)
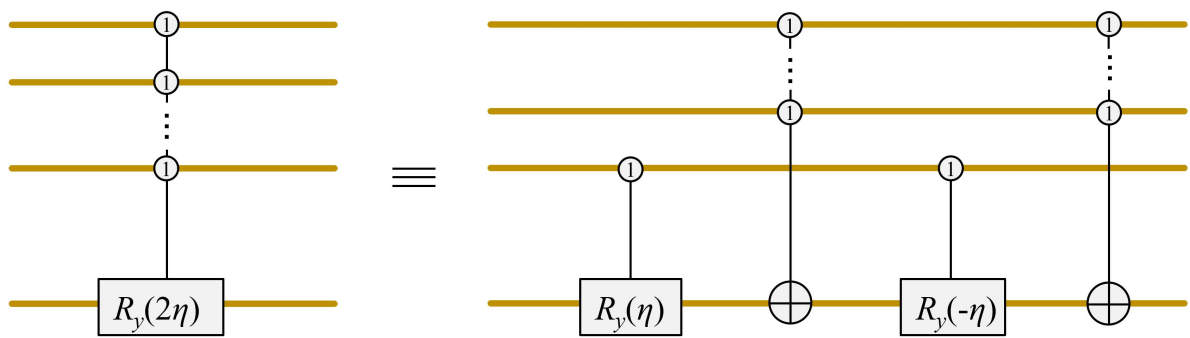

(b)

Figure 6. Circuit implementation of (a) controlled- $R_{y}(2 \eta)$ and (b) $R_{y}(2 \eta)$ operation with multiple control conditions.

In the circuit model of quantum computation, computation is steered by a sequence of unitary operations represented as quantum gates (presented earlier in Figure 7) that together with connecting wires act on one or more qubits to simultaneously affect each element of the superposition and generate massive parallel data processing [38]. In this model, the complexity and, conversely, the simplicity of executing a quantum algorithm is determined by the ability to concatenate and breakdown layers of the circuit in terms of basic or elementary quantum gates. In Figure 7, such complex layers (on the left) are decomposed in terms of the simpler NCT gate libraries (on the right), i.e., composed of NOT, CNOT, and Toffoli gates. In this manner, seemingly complex layers of a circuit can be decomposed into simpler ones as illustrated in Figure $7 \mathrm{c}$, where an $n$-controlled NOT gate is decomposed into $2(n-1)$ Toffoli gates as well as 1 CNOT gate, and 1 Toffoli gate can be further approximately simulated by 6 CNOT gates [39].
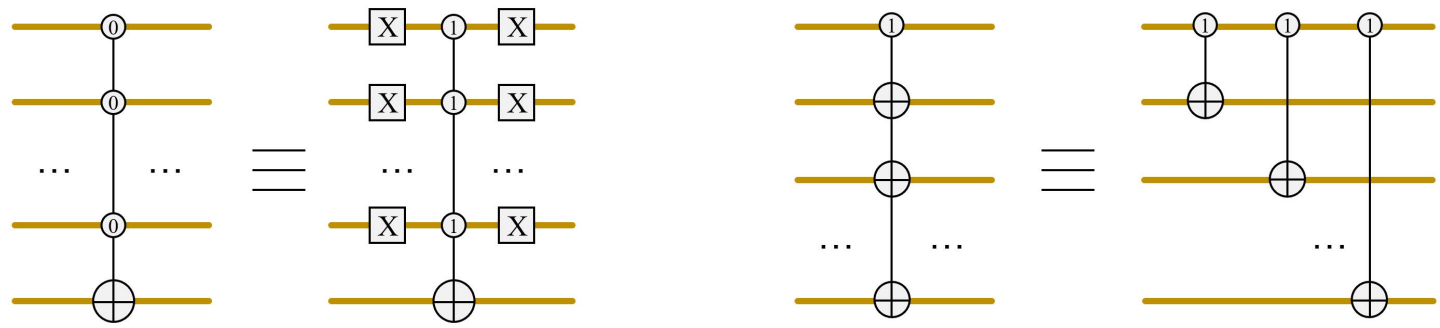

(a)

(b)
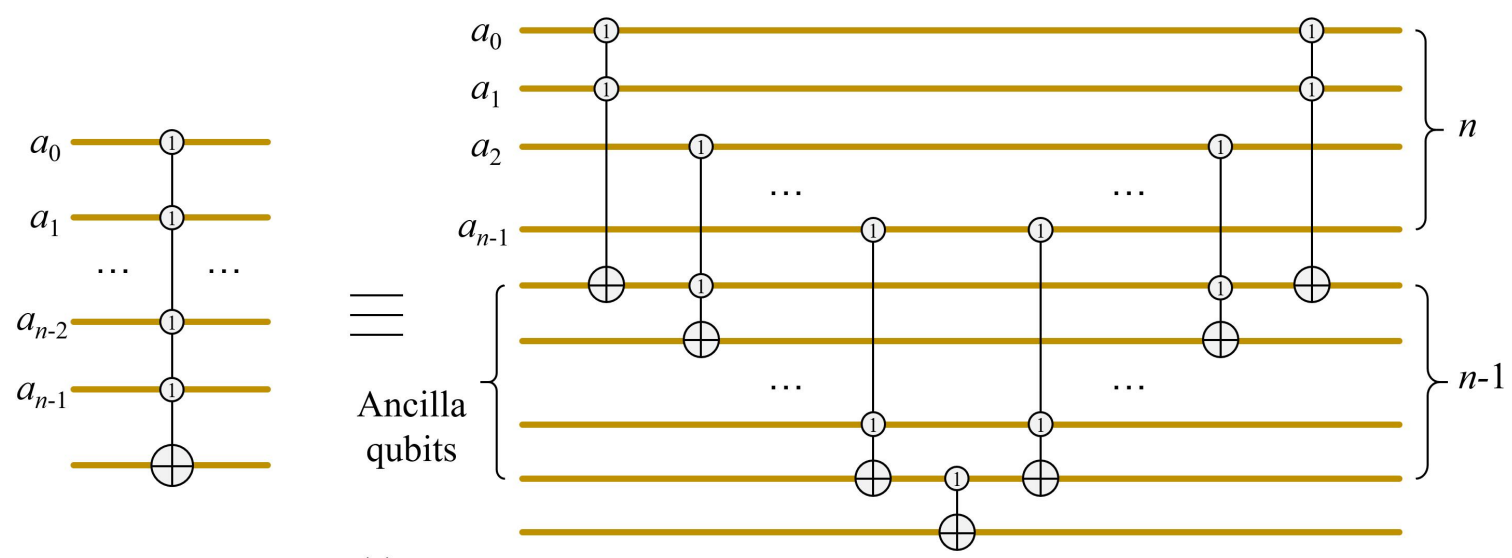

(c)

Figure 7. Decomposition of quantum circuits: (a) NOT gate controlled by a sequence of 0 conditions, (b) multiple NOT gates controlled by 1-condition, and (c) further decomposition of (a) into a network of basic gates. 


\section{Fusion of Quantum Emotions for Multi-Robots}

Emotion fusion entails the combination (i.e., mixture) of the emotions of multi-robots in a specified communication scenario or environment. The emotion fusion state of all robots (or part of them) can be controlled or changed using external stimulus.

We assume an enclosure (such as a room) containing $N$ robots, and each robot is identified by a number $(k=0,1,2, \cdots, N-1)$. To simplify things, we divide the enclosure into $2^{n} \times 2^{n}$ lattices $\left(N=2^{n} \times 2^{n}\right)$, as shown in Figure 8. Meanwhile, Figure 8a shows the multi-robots in a room, while the $X, Y$, and $Z$ axes constitute $H, W$, and $V$ planes. As an example, Figure $8 \mathbf{b}$ shows, the details of the $H$ plane where the rows and columns along the plane indicate the levels of the qubits encoding the emotions in the affected area. For instance, spatially the robots in the square "ABCD" can be presented by $\left|y_{1} y_{0} x_{1} x_{0}\right\rangle=$ $|1100\rangle$, while those in the square "AEFG" comprising of four robots can be simultaneously controlled by the qubit sequence $\left|y_{1} x_{1}\right\rangle=|10\rangle$. This illustrates that the more robots we want to control, the less qubits we need.

Therefore, the emotion fusion of these $N$ robots can be represented as

$$
\begin{gathered}
|\widetilde{E}(\xi)\rangle=\frac{1}{2^{n}} \sum_{k=0}^{2^{2 n}-1}\left(\cos \xi_{k}|0\rangle+\sin \xi_{k}|1\rangle\right) \otimes|k\rangle, \\
\xi_{k} \in\left[0, \frac{\pi}{2}\right], k=0,1, \cdots, 2^{2 n}-1,
\end{gathered}
$$

where $\otimes$ is the tensor product notation, $|k\rangle, k=0,1, \cdots, 2^{2 n}-1$, are $2^{2 n}$-D computational basis quantum states and $\xi=\left(\xi_{0}, \xi_{1}, \cdots, \xi_{2^{2 n}-1}\right)$ is the vector of angles encoding emotions. There are two parts in a typical scenario for multi-robot emotion representation: $\cos \xi_{k}|0\rangle+$ $\sin \xi_{k}|1\rangle$ encodes the emotional information and $|k\rangle$ is about the corresponding locations (i.e., the identity) of each robot in the scenario, respectively. Therefore, to facilitate the emotion fusion of these $N$ robots requires $2 n+1$ qubits, where $2 n$ qubits specify the robot's spatial location in the enclosure and the remaining one qubit is used to identify the emotional states of all robots, i.e., the controlled condition operations applied on the locations ( $\mathrm{Y}$ and $\mathrm{X}$ axes) are used to confine the present emotion to a targeted robot. In this manner, transformations on a multi-robot quantum atmosphere facilitate parallel manipulation (communication atmosphere is described as the psychological factor and feeling that can affect the behaviourial process and result in the space, which is usually obtained by fusing individual emotional states). Finally, the fused emotional state of many or multi-robots is a normalised state (i.e., similar to the one composed of intricacies of many humans in some environment (or predefined) scenario), i.e., $\||\widetilde{E}(\widetilde{\xi})\rangle \|=1$ as given by

$$
\||\widetilde{E}(\xi)\rangle \|=\frac{1}{2^{n}} \sqrt{\sum_{i=0}^{2^{2 n}-1}\left(\cos ^{2} \xi_{k}+\sin ^{2} \xi_{k}\right)}=1 .
$$

Next, we discuss how to realise the emotion fusion. Given a vector $\xi=\left(\xi_{0}, \xi_{1}, \cdots\right.$, $\left.\xi_{2^{2 n}-1}\right)$ of angles $\left(\xi_{k} \in\left[0, \frac{\pi}{2}\right], k=0,1, \cdots, 2^{2 n}-1\right)$, we turn quantum computers from the initialized state, $|0\rangle^{\otimes 2 n+1}$, to the quantum emotion state in Equation (13), composed of a polynomial number of simple gates.

First, using the two-dimensional identity matrix $I$ and the $2 n$ Hadamard matrices $H^{\otimes 2 n}$, the transform $\mathcal{H}=I \otimes H^{\otimes 2 n}$ applied on $|0\rangle^{\otimes 2 n+1}$ produces the state $|H\rangle$, presented as

$$
\mathcal{H}\left(|0\rangle^{\otimes 2 n+1}\right)=\frac{1}{2^{n}}|0\rangle \otimes \sum_{i=0}^{2^{2 n}-1}|i\rangle=|H\rangle .
$$

So far, we have formally separated the room into a $2^{n} \times 2^{n}$ lattices, i.e., each robot in the enclosure has a unique identification. Subsequently, we relate each robot a specified emotion. 


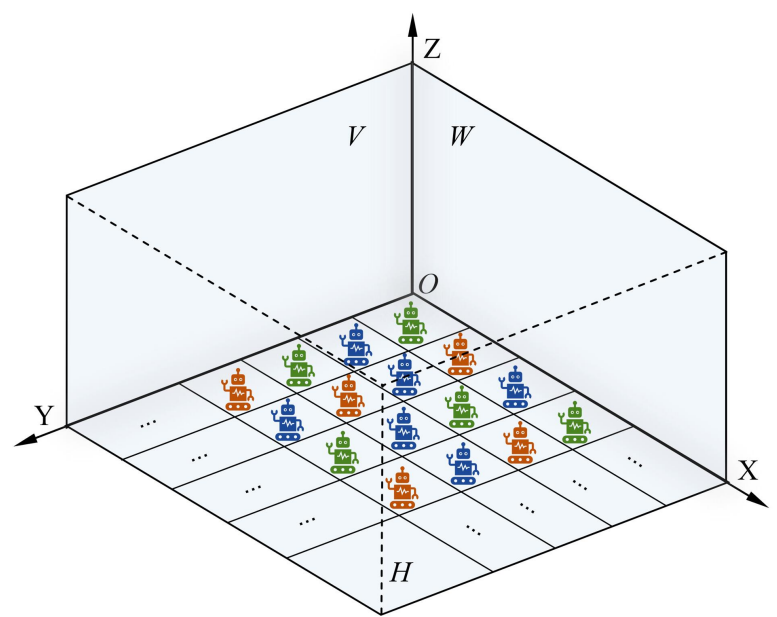

(a)

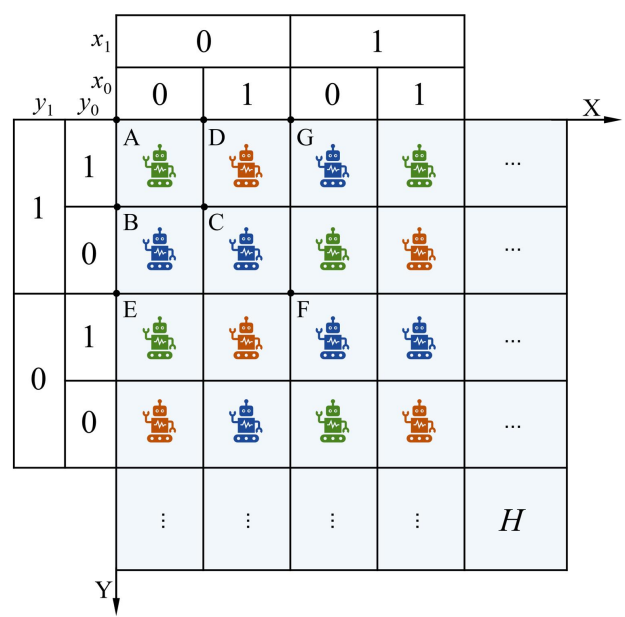

(b)

Figure 8. Illustration of (a) multi-robots in the same enclosure or environment (e.g., a room), and (b) the quantum binary demonstration of the location of each robot in the room.

The rotation matrices $R_{y}\left(2 \xi_{k}\right)$ as defined in Equation (2) and controlled-rotation matrices $R_{k}$ with $k=0,1, \cdots, 2^{2 n}-1$ are considered, i.e.,

$$
R_{k}=\left(I \otimes \sum_{j=0, j \neq k}^{2^{2 n}-1}|j\rangle\langle j|\right)+R_{y}\left(2 \xi_{k}\right) \otimes|k\rangle\langle k| .
$$

This controlled-rotation $R_{k}$ is a unitary matrix, since $R_{k} R_{k}^{+}=I^{\otimes 2 n+1}$. Meanwhile, applying $R_{S}$ and $R_{t} R_{S}$ on $|H\rangle$ produces:

$$
\begin{aligned}
R_{s}(|H\rangle) & =R_{s}\left(\frac{1}{2^{n}}|0\rangle \otimes \sum_{k=0}^{2^{2 n}-1}|k\rangle\right) \\
& =\frac{1}{2^{n}}\left[I|0\rangle \otimes\left(\sum_{k=0, k \neq s}^{2^{2 n}-1}|k\rangle\langle k|\right)\left(\sum_{k=0}^{2^{2 n}-1}|k\rangle\right)\right. \\
& \left.+R_{y}\left(2 \xi_{s}\right)|0\rangle \otimes|s\rangle\langle s|\left(\sum_{k=0}^{2^{2 n}-1}|k\rangle\right)\right] \\
& =\frac{1}{2^{n}}\left[|0\rangle \otimes\left(\sum_{k=0, k \neq s}^{2^{2 n}-1}|k\rangle\right)+\left(\cos \xi_{s}|0\rangle+\sin \xi_{s}|1\rangle\right) \otimes|s\rangle\right],
\end{aligned}
$$

and

$$
\begin{aligned}
R_{t} R_{s}|H\rangle & =R_{t}\left(R_{s}|H\rangle\right) \\
& =\frac{1}{2^{n}}\left[|0\rangle \otimes\left(\sum_{k=0, k \neq s, t}^{2^{2 n}-1}|k\rangle\right)+\left(\cos \xi_{s}|0\rangle+\sin \xi_{s}|1\rangle\right) \otimes|s\rangle\right. \\
& \left.+\left(\cos \xi_{t}|0\rangle+\sin \xi_{t}|1\rangle\right) \otimes|t\rangle\right] .
\end{aligned}
$$

In addition, it is clear from Equation (19) that

$$
\mathcal{R}|H\rangle=\left(\prod_{k=0}^{2^{2 n}-1} R_{k}\right)|H\rangle=|\widetilde{E}\rangle .
$$


Therefore, the unitary transform $\mathcal{P}=\mathcal{R} \mathcal{H}$ turns a quantum computing hardware from the initialized state $|0\rangle^{\otimes 2 n+1}$ to the emotion fusion state $|\widetilde{E}\rangle$. The total number of simple operations used to prepare the fusion emotion state is

$$
2 n+2^{2 n} \times\left(2^{2 n-1}-1+2^{2 n-1}-2\right)=2^{4 n}-3 \times 2^{2 n}+2 n .
$$

Consequently, the computational complexity for fusing the emotional states of multi-robots can be calculated as $O\left(2^{4 n}\right)$.

While the storage of an emotional state is accomplished during the preparation process, the measurement of the quantum emotion state produces a probability distribution that is used to retrieve the emotion. As presented earlier, measurement of a quantum state produces only one result, which is one entry in a set of basis vectors. Therefore, with only one quantum state, it is impossible to obtain information from that state; hence, a measurement process requires many identical quantum states and multiple measurement operations on these identical states recovers information about the quantum state [40] which in our case is information about the emotion of each robot in the form of a probability distribution.

As discussed earlier, transformations on a multi-robot quantum emotion facilitate parallel manipulation in each enclosure or setting. In the sequel, we illustrate the use of control conditions to confine the rotation operation to transform the emotion of each robot or multi-robots in an environment. Suppose an $8 \times 8$ enclosure to accommodate 64 robots composed of a lattice that is divided into 4 squares, i.e., 16 robots in each square shown in the top figure in Figure 9a). Further, we suppose that all the 16 robots in each square have the similar emotions that are encoded using the same colour. This is similar to a competitive match, where, for example, there are four teams and they each share some emotional states (e.g., expectation) and each team will also have emotions unique to them. After the match, the team's emotion will change according to the match result, e.g., the winner will be happy and the loser will be sad or even angry. To encode this and further explain the example, we further divide the lattice into an upper and lower half, which can be used to constrict the operation by focusing on $R_{y}(-\pi / 4)$ (using state " 1 " control condition) and $R_{y}(-\pi / 2)$ (using state " 0 " control condition) to the upper and lower halves of the plane as shown in in Figure 9b, using which we can transform the emotion to its opposite state on the emotion spectrum. Together with Equation (11), the bottom figure in Figure 9a illustrates the original and resultant emotion states (we only use the central $2 \times 2$ lattice, i.e., 4 robots, to show their locations and change in emotional states).

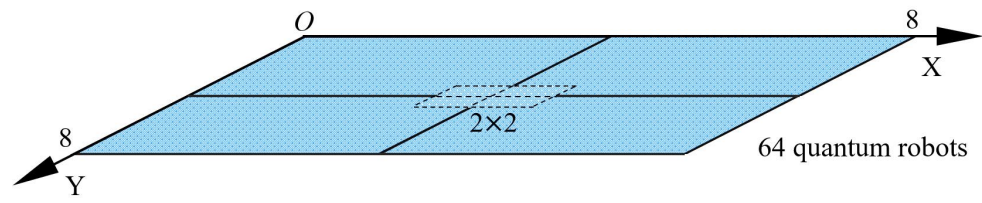

\begin{tabular}{|c|c|c|c|}
\hline $\begin{array}{c}\frac{3 \pi}{16} \\
100011\end{array}$ & $\begin{array}{c}\frac{\pi}{4} \\
100100\end{array}$ & $\begin{array}{c}\frac{\pi}{16} \\
100011\end{array}$ & $\begin{array}{c}\frac{\pi}{8} \\
100100\end{array}$ \\
\hline $3 \pi$ & $5 \pi$ & $\pi$ & $\pi$ \\
\hline 8 & $\overline{16}$ & $\overline{8}$ & $\overline{16}$ \\
\hline 011011 & 011100 & 011011 & 011100 \\
\hline
\end{tabular}

(a)

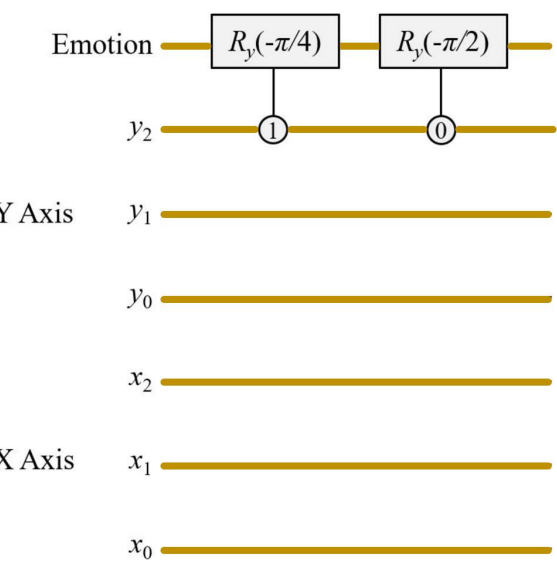

(b)

Figure 9. (a) Example showing an $8 \times 8$ lattice divided into four quadrants that each includes 16 robots with similar emotions and (b) circuit for transformation of their emotions.

Extracting and subsequently analysing the distributions of probability read outs resulting from measurement operations provides the information needed to retrieve a new emotion state. As mentioned in Section 2.1, the total probability of states $|0\rangle$ and $|1\rangle$ in an 
emotion state equals 1 . Therefore, when the $|0\rangle$ is measured, the probability distribution of the $k$ th robot can be recovered using

$$
\operatorname{Prob}(k)=2^{-2 n} \cos ^{2} \theta_{k} \text {. }
$$

Figure 10 presents the probability distribution for the fused emotion environment described earlier where (a) and (b) show the fused emotions prior to and after the transformation in Figure 9 (i.e., via the circuit in Figure 9b). These distributions provide a visual impact of the quantum rotation operation on the emotional states of each robot.

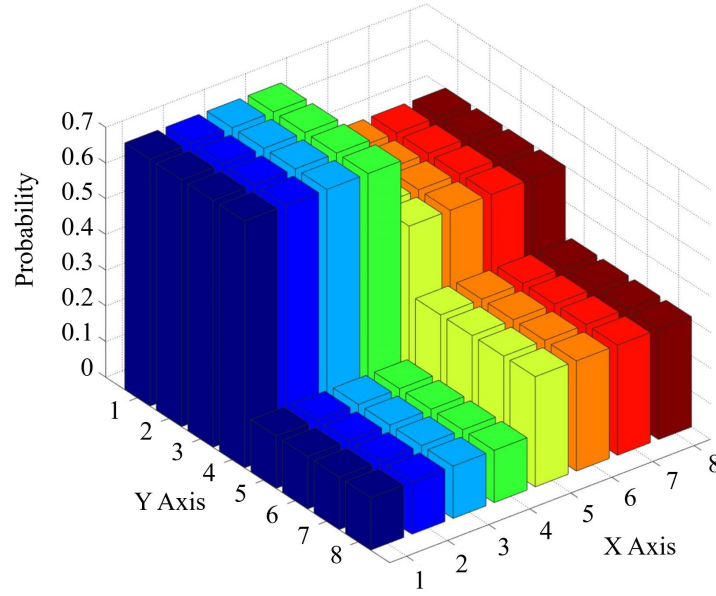

(a)

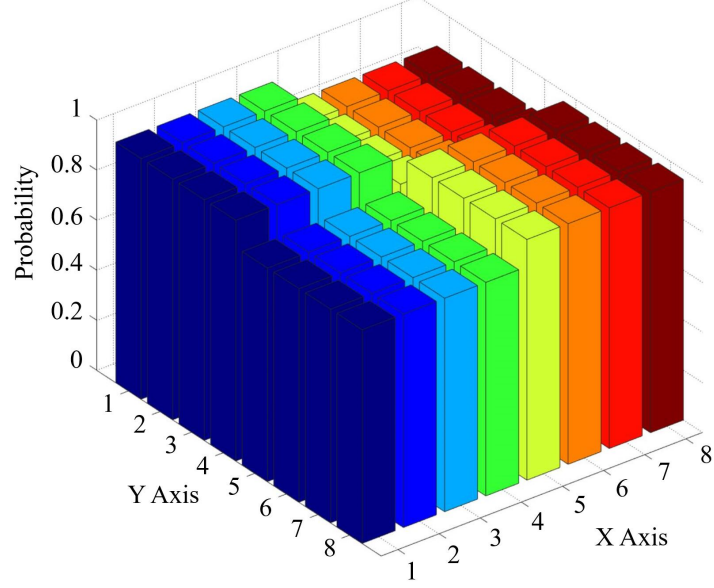

(b)

Figure 10. Probability distribution obtained from the emotion in Figure 9 where (a) shows distributions before and (b) that after the application of the quantum rotation operation.

While we have devoted a lot of attention to describing the potential use of quantum mechanics in affective computing, we would also like to reiterate that the 'quantumness' in these operations remains the focal point of any future quantum affective framework (QAC). This is especially useful for in stimulating the interest of practitioners with little or no background in quantum computing towards the pursuit of harnessing the potency of quantum computing to enhance traditional affective computing paradigms. In addition to the potential use of the proposed module in future quantum robotic systems, we emphasize two advantages that the proposed QAC paradigm offers us. First, referring to Equation (13), in discussing the emotion states of $N$ robots, we saw how the quantum superposition works. There, we used only one superposition qubit to encode the emotional states of all the $N$ robots and used the control functions to differentiate (or restrict) each robot to certain emotions. This reduces a lot of computing resources, especially, when a lot of robots are involved. In addition, as shown in Figure 9, and referring to the discussion in Section 2.1, quantum computation supports simultaneous evaluation of a function $f(x)$ for many values of $x$, which is referred to as quantum parallelism. Therefore, in the example shown in Figure 9, performing the rotation operation once can change the emotional state of all the robots in the system, and via the use of control conditions, two rotation operations could further divide the lattice into two parts, each with its own transformation. In this manner, the more controls a transformation has, the smaller the size of the area affected by it. However, such a transformation comes with additional costs in terms of the depth and the number of basic gates to confine the required circuit to a predetermined location [26,38].

\section{Concluding Remarks}

The massive amount of data that will be generated by future sensors combined with the rising complexity associated with controlling, planning, interacting, and reasoning systems, as well as the fact that future robots will operate in all kinds of environments, especially those to facilitate interactions with humans provide evidence of the huge computing 
demands of future robotic systems. This will be further compounded by the integration of internet of things (IoT) into robotics for advanced smart homes, assisted living, industrial IoT and other future technologies. Meanwhile, quantum systems are credited with astounding capabilities ascribed to its properties of entanglement, superposition and parallelism. Therefore, there is a consensus cum growing interest in harnessing quantum algorithms, quantum sensors, and quantum controls for important roles in future robotics and automated integrated systems.

While the early ideas of quantum robots are credited to Benioff [18], his ruminations did not posit the robot having an awareness nor does it envision the robot's ability to make decisions or measurements. Meanwhile, in Dong's work [29], which is more widely accepted by engineers, an alternative definition for quantum robots where their robots interaction with the external environment via sensing and information processing was considered for the first time. In this reasoning, a quantum robot is a mobile physical apparatus designed for using quantum effects of quantum systems, which can sense the environment and its own state, process quantum information and accomplish meaningful tasks. With such an engineering perspective, they formulated several fundamental components to compose a quantum robot's information acquisition and communication.

In an attempt to offer some modest contributions to the above discourse, our study explores the rudimentary processes for interpreting standard notations and operations in affective computing using properties of quantum computation. The main motivations are twofold: first, is to stimulate interest in microcosmic interpretation of emotions and affective computing in terms of the precepts of quantum mechanics. This is important considering that quantum technology provides veritable tools to model microcosmic phenomena that coalesce into colossally erratic registers that align with the unpredictable and oftentimes arbitrary or fluctuating nature of emotions which could be easily triggered, changed, or even extinguished depending on impulses, stimuli, etc. Second, in their quantum structure of robots, Dong et al. proposed the notion of multi-quantum computing units (MQCU), which exhibit marked similarities with the human brain. However, they did little to present a one-to-one correspondence between information processing in the two worlds. In this study, we advanced this effort by demonstrating how emotions in a quantum affective computing setting could be encoded and processed using quantum unitary operations and, by doing so, we surmised frameworks for communication between robots and their ability to make emotional decisions. While facile in its presentation, the study presents a first step in quantum affective computing (QAC) where many of the bewildering properties of quantum computing could be coalesced into distributions and descriptions of quantum emotion as well as the fusion of emotions in different robot-robot and robot-human interactive environments.

Among others, advantages of the proposed QAC include exploiting the parallel computing capability inherent to quantum computers to conserve computing resources as well as its offer of considerable increase in processing speed. Furthermore, the QAC framework facilitates the representation and fusion of multi-robot emotions in an enclosure.

Despite the enumerated potentials, in the long road to harnessing the potency of quantum computation for efficient affective computing, our ongoing and future work is motivated by the following considerations. First, the proposed QAC framework is supposed to be a dependent or interactive unit for exchange of information with other QCUs in the quantum robot system. Therefore, we may need to study how such a unit can communicate with others, including the necessary information acquisition, fusion, and distribution. In other Words, we should define the interface of QAC unit with other QCUs to make the affective processing of quantum robots and its corresponding response seamless and practical. Second, the four hypothetical devices of the QAC framework need to be further studied to ascertain which technologies are suited for processing quantum sensory information. For example, typical multi-robot or robot-human scenarios as well as changes in emotion states are influenced by external factors and stimuli. Following that, how to design the ES to extract the useful information from the external stimulus and encode it 
for further computing in the quantum system. Third, in the fusion of multi-robot emotion, it is known that multiple measurement operations required to recover an emotion state would impose additional computing costs. This must be carefully studied for efficiency. Ancilla-driven and measurement-based quantum computation [35] are viable alternatives to the circuit-model of quantum computation that have been considered in similar quantum readers. Consequently, it is expedient to study required refinements for their integration to help provide required trade-offs between accuracy and computational cost.

As argued in [41], to build a universal emotion space model with robust emotion recognition and expression systems, it is necessary to discriminate the different types of emotion and establish some basic mode of emotions. However, emotions are predilections that even in humans vary from person to person. They permeate, interact, and transition with each other, often with quite complex and frequent changes. In this regard, quantifying an emotion requires adequate emotion dataset as well as a large amount of computing overhead. Consequently, cross-disciplinary techniques, such as data mining and machine learning, must be considered to facilitate the robot's capability to have better multi-robot as well as human-robot interaction (HRI). Similarly, an important objective of emotional space modelling is to support the development of emotional robots and the design of humanrobot emotional interaction systems [42]. Therefore, in order to realise practicable and computationally efficient emotional models, effective HRI ingrained with robust emotion space models and standards (i.e., technical, and ethical) must be considered. Furthermore, extensive models for multimodal emotion fusion, communication atmosphere, intention understanding, and computational systems of emotions, etc., that can meet the personalised and non-personalised user needs are required for the development of emotional robots.

Author Contributions: Conceptualization, F.Y. and A.M.I.; methodology, F.Y. and A.M.I.; software, F.Y.; validation, F.Y., K.H.; formal analysis, K.H.; investigation, F.Y.; resources, F.Y. and A.M.I.; data curation, A.M.I. and K.H.; writing-original draft preparation, F.Y. and A.M.I.; writing-review and editing, F.Y., A.M.I. and K.H.; visualization, A.M.I.; supervision, K.H.; project administration, F.Y.; funding acquisition, A.M.I. All authors have read and agreed to the published version of the manuscript.

Funding: This study is sponsored by the Prince Sattam Bin Abdulaziz University, Saudi Arabia via the Deanship for Scientific Research funding for the Advanced Computational Intelligence and Intelligent Systems Engineering (ACIISE) Research Group Project Number 2020/01/12173.

Conflicts of Interest: The authors declare no conflict of interest.

\section{References}

1. Calvo, R.; D'Mello, S.; Gratch, J.; Kappas, A. The Oxford Handbook of Affective Computing; Oxford University Press: New York, NY, USA, 1997.

2. James, W. What is an emotion? Mind 1884, os-IX, 188-205. [CrossRef]

3. Picard, R. Affective Computing; The MIT Press: Cambridge, UK, 1997.

4. Poria, S.; Cambria, E.; Bajpai, R.; Hussain, A. A review of affective computing: From unimodal analysis to multimodal fusion. Inf. Fusion 2017, 37, 98-125. [CrossRef]

5. Guo, F.; Li, F.; Lv, W.; Liu, L.; Duffy, V. Bibliometric analysis of affective computing researches during 1999-2018. Int. J. Hum. Comput. Interact. 2020, 36, 801-814. [CrossRef]

6. Muñoz-Saavedra, L.; Luna-Perejón, F.; Civit-Masot, J.; Miró-Amarante, L.; Civit, A.; Domínguez-Morales, M. Affective state assistant for helping users with cognition disabilities using neural networks. Electronics 2020, 9, 1843. [CrossRef]

7. Hoorn, J. The robot brain server: Design of a human-artificial systems partnership. Adv. Intell. Syst. Comput. 2018, 722, 531-536.

8. Hoorn, J.; Ho, J. Robot affect: The amygdala as Bloch sphere. arXiv 2019, arXiv:1911.12128v2.

9. Aerts, D.; Melucci, M.; Bianchi, M.; Sozzo, S.; Veloz, T. Quantum structures in computer science: Language, semantics, retrieval. Theor. Comput. Sci. 2018, 752, 1-4. [CrossRef]

10. Nielsen, M.; Chuang, I. Quantum Computation and Quantum Information; Cambridge University Press: Cambridge, UK, 2000.

11. Schwartz, J.; Stapp, H.; Beauregard, M. Quantum physics in neuroscience and psychology: A neurophysical model of mind-brain interaction. Philos. Trans. R. Soc. B 2005, 360, 1309-1327. [CrossRef]

12. Aerts, D.; Sozzo, S.; Veloz, T. Quantum Structure in Cognition and the Foundations of Human Reasoning. Int. J. Theor. Phys. 2015, 54, 4557-4569. [CrossRef]

13. Aerts, D. Quantum structure in cognition. J. Math. Psychol. 2009, 53, 314-348. [CrossRef] 
14. Narens, L. On replacing "quantum thinking" with counterfactual reasoning. Context. Quantum Phys. Psychol. 2016, 309-324. [CrossRef]

15. Lukac, M.; Perkowski, M. Quantum mechanical model of emotional robot behaviors. In Proceedings of the 37th International Symposium on Multiple-Valued Logic, Oslo, Norway, 13-16 May 2007.

16. Raghuvanshi, A.; Perkowski, M. Fuzzy quantum circuits to model emotional behaviors of humanoid robots. In Proceedings of the IEEE Congress on Evolutionary Computation, Barcelona, Spain, 18-23 July 2010.

17. Yan, F.; Iliyasu, A.; Jiao, S.; Yang, H. Quantum structure for modelling emotion space of robots. Appl. Sci. 2019, 9, 3351. [CrossRef]

18. Benioff, P. Some foundational aspects of quantum computers and quantum robots. Superlattices Microstruct. 1998, 23, 407-417. [CrossRef]

19. Biamonte, J.; Wittek, P. Quantum machine learning. Nature 2017, 549, 195-202. [CrossRef]

20. Dunjko, V.; Briegel, H. Machine learning \& artificial intelligence in the quantum domain: A review of recent progress. Rep. Prog. Phys. 2018, 81, 074001.

21. Yan, F.; Venegas-Andraca, S. Quantum Image Processing; Springer: Berlin, Germany, 2020.

22. Benioff, P. Quantum robots and environments. Phys. Rev. A 1998, 58, 893-904. [CrossRef]

23. Cañamero, D. A hormonal model of emotions for behavior control. In Proceedings of the 4th European Conference on Artificial Life, Brighton, UK, 28-31 July 1997.

24. Hamilton, C. On the Possibility of Robots Having Emotions. Ph.D. Thesis, Georgia State University, Atlanta, GA, USA, 2014.

25. Petschnigg, C.; Brandstötter, M.; Pichler, H.; Hofbaur, M.; Dieber, B. Quatnum computation in robotic science and applications. In Proceedings of the IEEE International Conference on Robotics and Automation, Montreal, QC, Canada, 20-24 May 2019; pp. 803-810.

26. Iliyasu, A.; Le, P.; Dong, F.; Hirota, K. Watermarking and authentication of quantum images based on restricted geometric transformations. Inf. Sci. 2012, 186, 126-149. [CrossRef]

27. Yao, X.; Wang, H.; Liao, Z.; Chen, M.; Pan, J.; Li, J.; Zhang, K.; Lin, X.; Wang, Z.; Luo, Z. et al. Quantum image processing and its application to edge detection: Theory and experiment. Phys. Rev. X 2017, 7, 031041. [CrossRef]

28. Yan, F.; Iliyasu, A.; Khan, A.; Yang, H. Measurements-based moving target detection in quantum video. Int. J. Theor. Phys. 2016, 55, 2162-2173. [CrossRef]

29. Dong, D.; Chen, C.; Zhang, C.; Chen, Z. Quantum robot: Structure, algorithms and applications. Robotica 2006, $24,513-521$. [CrossRef]

30. Brennen, G.; Song, D.; Williams, C. Quantum computer architecture using nonlocal interactions. Phys. Rev. A 2003, 678, 050302. [CrossRef]

31. Liu, Z.; Wu, M.; Li, D.; Chen, L.; Dong, F.; Yamazaki, Y.; Hirota, K. Concept of fuzzy atmosfield for representing communication atmosphere and its application to humans-robots interaction. J. Adv. Comput. Intell. Intell. Inform. 2013, 7, 3-17. [CrossRef]

32. Kallias, G.; Devlin, E.; Christides, C.; Niarchos, D. High $T_{C}$ SQUID sensor system for non-destructive evaluation. Sens. Actuators 2000, 85, 239-243. [CrossRef]

33. Behet, M.; Bekaert, J.; Boeck, J.; Borghs, G. In $A s / A l_{0.2} G a_{0.8} S b$ quantum well Hall effect sensors. Sens. Actuators 2000, 81, 13-17. [CrossRef]

34. Dong, D.; Petersen, I. Quantum control theory and applications: A Survey. IET Control Theory Appl. 2010, 4, 2651-2671. [CrossRef]

35. Iliyasu, A.; Le, P.; Dong, F.; Hirota, K. A framework for representing and producing movies on quantum computers. Int. J. Quantum Inf. 2011, 9, 1459-1497. [CrossRef]

36. Russell, J.; Fernandez-Dols, J. Reading Emotion from and into Faces-The Psychology of Facial Expression; Cambridge University Press: New York, NY, USA, 1997.

37. Colman, A. A Dictionary of Psychology; Oxford University Press: New York, NY, USA, 2015.

38. Iliyasu, A. Towards Realising Secure and Efficient Image and Video Processing Applications on Quantum Computers. Entropy 2013, 15, 2874-2974. [CrossRef]

39. Yan, F.; Iliyasu, A.; Venegas-Andraca, S. A survey of quantum image representations. Quantum Inf. Process. 2016, 15, 1-35. [CrossRef]

40. Yan, F.; Li, N.; Hirota, K. QHSL: A quantum hue, saturation, and lightness color model. arXiv 2020, arXiv:2007.09659.

41. Yan, F.; Iliyasu, A.; Hirota, K. Emotion Space Modelling for Social Robots. Eng. Appl. Art. Intell. 2021, 100, 1-25.

42. Marsella, S.; Gratch, J.; Petta, P. Computational models of emotion. A Bluepr. Affect. Comput. Sourceb. Man. 2010, 11, 21-46. 